\title{
Thumbnail Images Using Resampling Method
}

\author{
Lavanya Digumarthy ${ }^{1}$, CH.Sri Giri ${ }^{2}$, Dr.V.Sailaja ${ }^{3}$ \\ Mtech Student DECS, ECE Godavari Institute of Engineering Rajahmundry, India \\ ${ }^{2}$ Asst.Professor Electronics and Communication Engineering Godavari Institute of Engineering Rajahmundry, \\ India \\ ${ }^{3}$ Professor Electronics and Communication Engineering Godavari Institute of Engineering Rajahmundry, India
}

Abstract: A standard image thumbnail is generated by filtering and sub sampling when the blur and noise of an original image is lost since the standard thumbnails do not distinguish between high quality and low quality originals. In this paper an efficient algorithm with a blur - generating component and a noise generating component preserves the local blur and the noise of the originals. The new thumbnails are more representative of their originals for blurry images. The noise generating component improves the results for noisy images but degrades the results for textured images. The decision to use the noise component of the new thumbnails should base on testing with the particular image mix expected for the application.

Keywords: Standard thumbnails, image quality, noise modelling

\section{Introduction}

Filtering and sub sampling prevents aliasing and preserves image composition and loses its image quality .Both sharp and blurry original appear sharp in the thumbnails, and both clean and noisy originals appear clean in the thumbnails This leads to errors and inefficiencies during image selection based on the thumbnails rather than the high resolution originals.

The standard thumbnails introduced in [3] do not modify the image composition but better reflects the image quality of the originals. The New thumbnails provide a quick, natural way for users to identify images of good quality at the same time that they select images with desired subject matter. The new thumbnails are natural to interpret; there is no learning necessary to understand the blur and noise in the new thumbnails. The alternate approach of automatic image ranking by quality is extremely difficult because the knowledge about the subjects of interest resides with the user, not with the image .In the new thumbnails the user can check whether the subject of interest is in focus.

Several recent papers offer interesting, nontraditional algorithm for reducing image size. None of the algorithms address image quality and all of them modify image composition while resizing. The paper addressing image with different types of contents such as webpages, documents and photographs. This paper develops image previews that address image quality to use iterative gradient field computations to accentuate selected image features.

There are many applications for the new thumbnails. A digital camera display could provide a quick look at the image quality of the originals. If the images are of low quality, the user could immediately take another picture. In addition, current photo browsing applications and operating systems both provide image thumbnails during browsing. Thumbnails are often generated on embedded devices with limited computational power, or they are generated in batch in large numbers. To find wide spread use, quality-preserving thumbnails need efficient computation.

This paper is organized as follows section IV describes the new algorithm for space-varying blur estimation and blur generation for the new thumbnails, and section $\mathrm{V}$ describes the fast method for preserving noise in the thumbnails. Section VI describes subjective experiments testing the thumbnails against standard thumbnails .section VII ends finally with the conclusion.

\section{Image Model And Formulation}

The derivation below use 1-D column-stacked vector notation to simplify the representation as vector

$\mathrm{X}$, is

$$
\boldsymbol{x}=B \boldsymbol{s}+\boldsymbol{\eta} \text {. }
$$

In this equation the vector $\mathrm{S}$ represents an ideal image captured with infinite depth of field. The matrix $\mathrm{B}$ represents a space-varying blur corresponding to unintended blurs such as camera shake, motion blur as well as intended depth of blur and $\mathrm{n}$ represents an additive, perhaps correlated, noise. .Digital photographs taken under ideal conditions have no unintended blur or noise in this case the noise $\boldsymbol{\eta}=\mathbf{O} \cdot$ but the matrix B is not necessarily the identity, since it is still represents the space-varying blur due to limited depth of field together 
with objects of different distances. Only in the special case of infinite depth of field does B=I and therefore $\mathrm{X}=\mathrm{S}$.

The goal is to recover $\mathrm{S}$ from $\mathrm{X}$ generates a low resolution representative thumbnail $\mathbf{t}_{\mathbf{r}}$, not the exact reconstruction of high resolution S.For exaample our solution works well with both shake and defocus blurs,by applying an appropriate space varying blur.The exact form of the applied blur kernel is not critical.

The new thumbnail is generated by starting first with a standard thumbnail generated by filtering and subsampling, $\mathbf{t}_{\mathbf{s}}$, which is blur and noise free even for distorted input images. To this standard thumbnail,blur and noise are applied to correspond to the blur and noise in the original

$$
\boldsymbol{t}_{r}=\boldsymbol{t}_{b}+\boldsymbol{\rho}=B_{t} \boldsymbol{t}_{s}+\boldsymbol{\rho}_{1}
$$

\section{New Companding Algorithm}

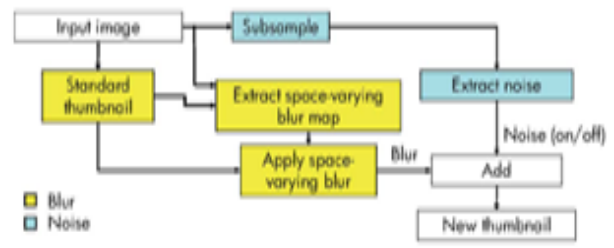

Fig.1.To generates the new thumbnail, the standard thumbnail is modified with a space-varying blur and an estimated noise is added.

The standard thumbnails is given by

\section{Standard Thumbnails}

$$
\boldsymbol{t}_{s}=T_{s} \boldsymbol{x}=S A \boldsymbol{x}
$$

Where it combines filtering and subsampling.Expanding (2) using the image modelling (1) results in

$$
\boldsymbol{t}_{\mathrm{s}}=S(\boldsymbol{A B \boldsymbol { s }}+\boldsymbol{A \boldsymbol { \eta }}) .
$$

The bandwidth of the blur is broader than the bandwidth of the antialiasing filter for typical subsampling factorsThus in (4) noise next,antialising filter applied result in output filtered noise variance much lower than the input variance.The case of a $\mathrm{k} \mathrm{X} \mathrm{k}$ boxcar filter,for a subsampling factor $\mathrm{k}$ is particularly easy to analyze.If the input noise is uncorrelated,the output noise variance will be reduced by a factor of $1 / \mathrm{k}^{\wedge} 2$.

The Mean square error(MSE) between a distortion image and undistorted image is proportional to the square of the Eucledian norm between the images interpreted as vectors.

The distorted image may be expressed as the addition of the two vectors to the original image, given by

$$
\boldsymbol{x}_{i j}=\boldsymbol{s}+\left(B_{i} \boldsymbol{s}-\boldsymbol{s}\right)+\boldsymbol{\eta}_{j}=B_{i} \boldsymbol{s}+\boldsymbol{\eta}_{j} .
$$

\section{Local Blur Computation}

Many prior methods for image blur determination provides global blur metrics that assess the overall quality of an image. The approach to provide local blur for detecting edges is given in [15].The blur map is determined without the type of identification of the type of the blur.

The low pass images are created by convolving the standard thumbnail with Gaussian kernels of different variances is given as

$$
\boldsymbol{l}_{\sigma_{j}}=g_{\sigma_{j}} * \boldsymbol{t}_{s} \text {. }
$$

The number of scale space images determines how finely the blur can be represented but the algorithm is insensitive to the number of scales. 


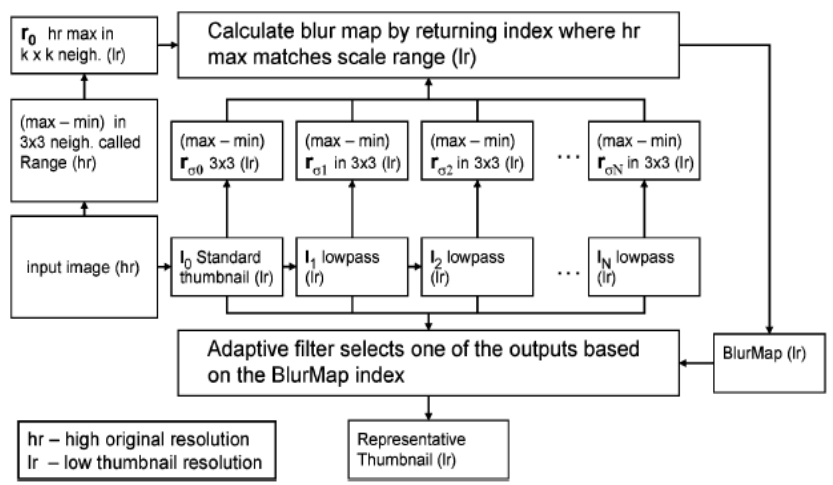

Fig.2.Blur Process involves comparing subsample high resolution pixel ranges in a scale space expansion.

The blur map shown in Fig.1. And Fig.2.is computed by comparing local range images. The generation of the blurred thumbnail images is linear in the number of thumbnail pixels.

A. Introduction and problem Formulation

\section{Noise Computation}

The noise generation developed by borrowing directly from image denoising, works well but it slow. Adding random jitter to the sub sampling breaks up potential moiré from any residual image textures that may erroneously appear in the noise image. The total blur and noise computation for converting an original image takes $0.14 \mathrm{~s}$ instead of the original $2.25 \mathrm{~s}$ on a $2-\mathrm{GHZ}$ processor and 1GB Ram.

The results in image model is given by

$$
\boldsymbol{x}=\boldsymbol{s}+\boldsymbol{\eta}
$$

Where $\mathrm{x}$ is a noisy originalimage, $\mathrm{s}$ is an idealimage.

$\mathrm{S}=$ undistorted image and $\mathrm{n}=$ noise

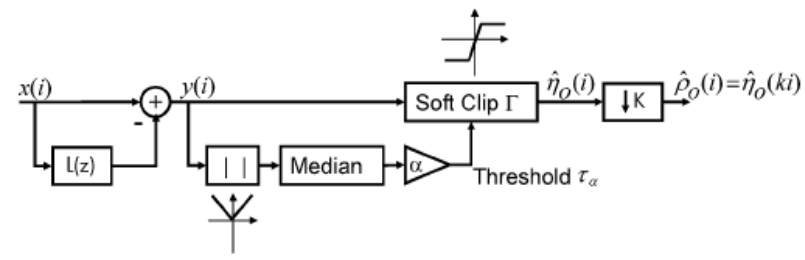

Fig.3.Block diagram for noise preservation based on denoising.

\section{B. Solution Using Denoising and Sub sampling}

The Threshold is determined by first robustly estimation noise standard deviation The computation of the threshold requires the high resolution signal. There are enough pixels even at the thumbnail resolution, for an effective determination of the noise variance of the original signal.

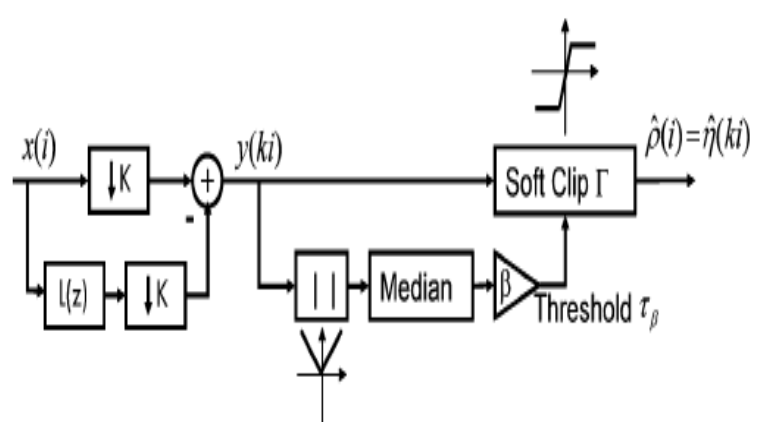

Fig.4.Block diagram for the noise generation algorithm.

The difficulties of the noise generating algorithm are in distinguishing between high frequency textures from noise. The nonlinear soft clip is memory less, it commutes with subsample. Even though there are 
nonlinear steps in the original algorithm for determining the threshold value and also for applying the memory less nonlinear operation.

The algorithm was first tested informally with several images and it was found effective for blurry and noisy images. There were however, differences between the standard and new thumbnails for textured images. By turning off the noise processing, corresponding to an additive noise.

The noise term was found to account for the differences in the textured images. This is due to the currently used noise algorithm, which does not always distinguish between image noise and texture, both of which contains high spatial frequencies are in the input image that results in more blur distortion. The faster increase of MSE along the blur axis confirming that the noise MSE in this case is image independent.

\section{Performance Simulation}

The input image is $620 \times 792$ pixels and the size is $25.4 \mathrm{~KB}$ by applying standard thumbnail and the new thumbnails the simulations are taken blur versus Mean square Error (MSE) and blur versus PSNR.

At both resolutions, users find that the new thumbnails are (1) Better represents the blurry and noisy images (2) They are not significantly different than standard thumbnails for the clean images. For most of the textured images, however, the users prefer the standard thumbnails.

Thumbnail treatments were positioned to appear on the right and left side randomly. Image samples were presented to each observer in a different random order. This technique distributes any start up effects over different samples.
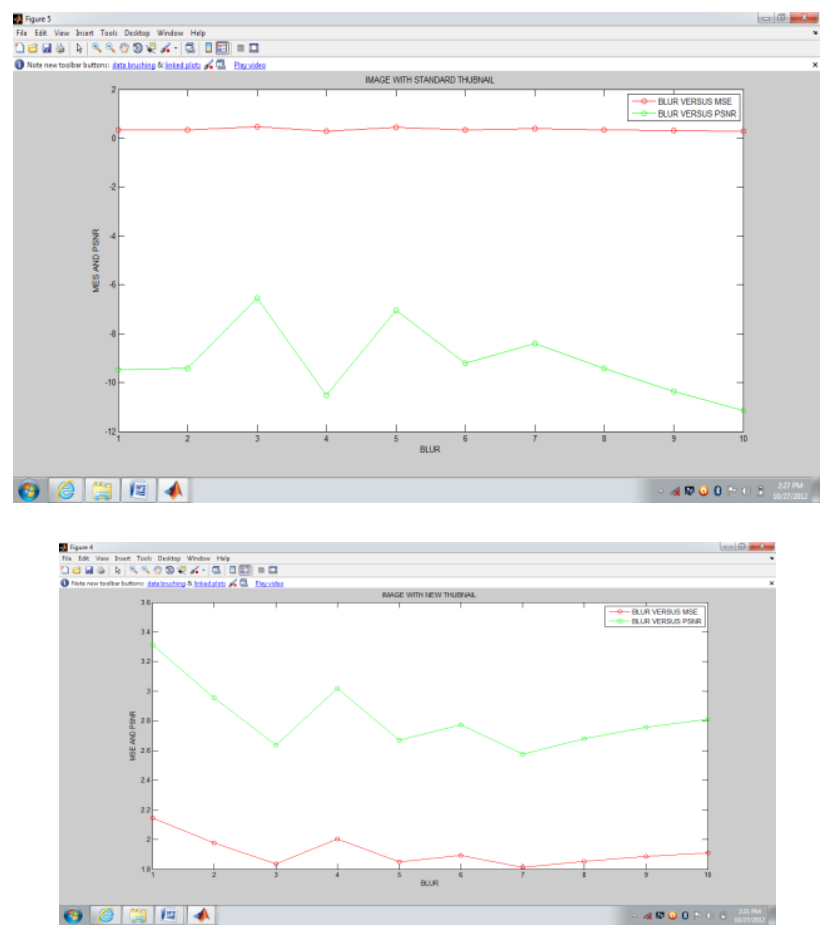

The total elapsed time that is blurring and noise time for converting an original image into new thumbnail is 194.760236seconds.

The complexity of the blur computation is composed of two parts (1) the complexity of generating the standard thumbnail and extracting features which are both linear in the number of the original image pixels since both computations compromise local computations in small constant sized neighborhoods. (2) The generation of the blurred thumbnail images is linear in the number of thumbnail pixels. Thus the overall complexity is linear in the number of original pixels.

\section{Conclusion}

A new image resizing method that preserves the image blur and the image noise .The subjective evaluation of the thumbnail shows that the blur component of the algorithm is robust and it may always be used with improved results. Equal number of the different image categories was used to better test the different cases. 


\section{Acknowledgements}

I grateful thanks to management of, Godavari Institute of technology, for him stimulating guidance and generous assistance.

\section{References}

[1] A.K.Jain Fundamentals of Digital Image Processing.Englewood cliffs NJ; Prentice-Hall,1989.

[2] A.Munoz,T.Blu,and M.Unser,"Least-squares image resizing using finite differences",IEEE Trans.Image Process,2001.

[3] R.Samadani,S.Lim, and D.Tretter, "Representative image thumbnails for good browsing,"in Proc.IEEE Int.Conf.Image Processing,sep2007,vol.II

[4] Y.Ke,X.Tang,and F.Jing, “ The design of high-level features for photo quality assessment,"in IEEE Computer Society Conf.Computer Visionand Pattern Recognition,2006,vol.1.

[5] A.Wondruff,A.Faulring,R.Roshenholtz,J.Morrison,andP.Prolli, "Using thumbnails to search the web,"in Proc.CHI,Apr.2001.

[6] B.Suh,H.Ling,B.Bederson,and .Jacobs,S.Wink “Automatic thumbnails cropping and its effectiveness," Proc.16th Annu.ACM,Dec.2003.

[7] K.Berkner,E.Schwartz,and C.Marle,L.Karamin "SmartNails:Display-and the image-independent thumbnails," in Proc.SPIE Document Recognition and Retrieval XI,Dec.2003.

[8] S.Avidan and A.Shamir, "Seam carving for content-aware image resizing,"ACM Symp.User In Trans.Graphics,vol.26,2007.

[9] L.Wan,W.Feng,Z.Lin,T.Wong, ndT.Ebriamian Z.Liu, "Perceptual image preview,”,oct.2008. 